\title{
Simulation of PEP-II Accelerator Backgrounds Using TURTLE
}

Contributed to Particle Accelerator Conference (PAC 05), 05/16/2005--5/20/2005, Knoxville, Tennessee 


\title{
SIMULATION OF PEP-II ACCELERATOR BACKGROUNDS USING TURTLE
}

\author{
R. Barlow, Manchester University, Manchester, UK \\ W. Kozanecki, DAPNIA-SPP, CEA-Saclay, 91191 Gif-sur-Yvette, France \\ P. Roudeau and A. Stocchi, LAL, 91898 Orsay, France \\ T. Fieguth, Stanford Linear Accelerator, Stanford CA 94309 USA* $^{*}$ \\ S. Majewski, Stanford University, Stanford CA 94305-4060 USA
}

\begin{abstract}
We present studies of accelerator-induced backgrounds in the BaBar detector at the SLAC B-Factory, carried out using LPTURTLE, a modified version of the DECAY TURTLE simulation package. Lost-particle backgrounds in PEP-II are dominated by a combination of beam-gas bremstrahlung, beam-gas Coulomb scattering, radiativeBhabha events and beam-beam blow-up. The radiation damage and detector occupancy caused by the associated electromagnetic shower debris can limit the usable luminosity. In order to understand and mitigate such backgrounds, we have performed a full programme of beamgas and luminosity-background simulations, that include the effects of the detector solenoidal field, detailed modelling of limiting apertures in both collider rings, and optimization of the betatron collimation scheme in the presence of large transverse tails.
\end{abstract}

\section{INTRODUCTION}

PEP-II [1] has reached $9.2 \times 10^{33} \mathrm{~cm}^{-2} \mathrm{~s}^{-1}$ with currents of $1.55 \mathrm{~A}$ in the High Energy $\left(e^{-}\right)$Ring (HER) and $2.45 \mathrm{~A}$ in the Low Energy $\left(e^{+}\right)$Ring (LER). High luminosity brings high backgrounds, increasing the readout time of the BaBar detector [2], worsening resolution, and causing radiation damage. With plans to increase the luminosity further, it became important to understand and mitigate these backgrounds, as done the design and commissioning. LPTURTLE has been a useful tool in this work.

The TURTLE ('Trace Unlimited Rays Through Lumped Elements") single-particle transport program was extended to include the decays of unstable particles (DECAY TURTLE), and to the study of particles lost through beam-gas collisions (LPTURTLE). A more extensive description of recent developments in the history of this long-lived program is presented at this conference [3].

\section{BEAM-GAS BACKGROUNDS}

The important processes are Coulomb Scattering and Bremsstrahlung, where the electron loses energy to form a photon. Photons give background only if produced close to or inside the interaction region (IR): scattered electrons may go a long way round the ring before hitting a wall.

Both processes have a pole (for zero degree scattering and zero energy photon emission). One has to apply a cut

\footnotetext{
* Work supported by US DOE contract DE-AC02-76SF00515
}

off, an angle or energy so tiny scattering does not throw the particle off orbit to the extent that it is lost. The simulation must concentrate (importance sampling) on scatters which produce significant backgrounds. Investigation showed that the scattering could be divided into ranges in the scattering parameter ( $\theta$ for Coulomb Scattering and $x_{\gamma}=E_{\gamma} / E_{\text {beam }}$ for Bremsstrahlung) and $z$, the point along the ring at which the scatter occurred. Depending on the ring and the type of scattering, between 5 and 8 ranges were found necessary. For very small scatters all particles survive. For small scatters, a particle survives unless the scatter occurs in certain ranges of $z$. There is an intermediate range for which the whole length has to be considered, and for large scatters, only interactions near the IP matter as the particle is lost shortly after the scatter. 100,000 particles were tracked in each range.
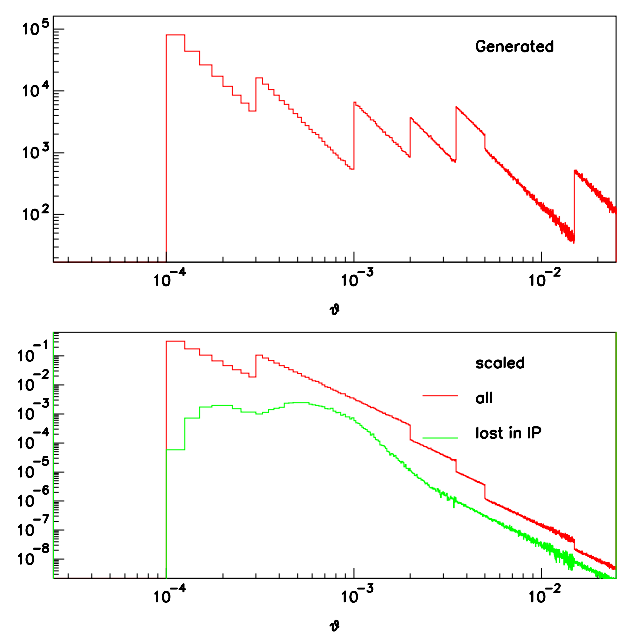

Figure 1: Sampling strategy for HER Coulomb scattering. The $x$ axis shows the scattering parameter and the $y$ axis the number of events(top) and the number of scattered particles per bunch crossing per $\theta$ bin (bottom).

This is illustrated in Fig. 1. The top plot shows the angles generated for Coulomb scattering in the HER: the sawtooth sections show the different regions of $\theta$ chosen. In the bottom plot the upper curve shows the top plot after scaling by a factor to give the predicted distribution of particle scatters. The second and third ranges in $\theta$ were generated over the full $z$ range but in other ranges of $\theta$ only selected ranges of $z$ were generated, and the scaled number 
of events is smaller. The lower curve shows the distribution in $\theta$ for particles which hit the wall near the interaction point (IP). There are no breaks in the lower curve, showing that the scatters not sampled are in regions of $z$ which never produce a particle that reaches the IP. The curves for Bremsstrahlung, and for the LER, are similar.

Fig. 2 shows the near-IR loss rates in the HER. The large loss around $5 \mathrm{~m}$ upstream, in both the upper and lower quadrants, is associated with Coulomb scattering, and is caused by betatron tails at large transverse amplitude in the final-doublet quadrupoles. In contrast, bremsstrahlung contributes most of the primary hits inside the detector, mostly in the horizontal plane (East and West quadrants). Here energy-degraded electrons are overbent by the combination of the last soft bend in the incoming straight, the final doublet, and the beam-separation dipoles located $20 \mathrm{~cm}$ from the IP. The magnetic (as opposed to crossing-angle) beam-separation scheme is one of the fundamental reasons why machine backgrounds are very different in the PEP-II and KEKB $B$-Factories.

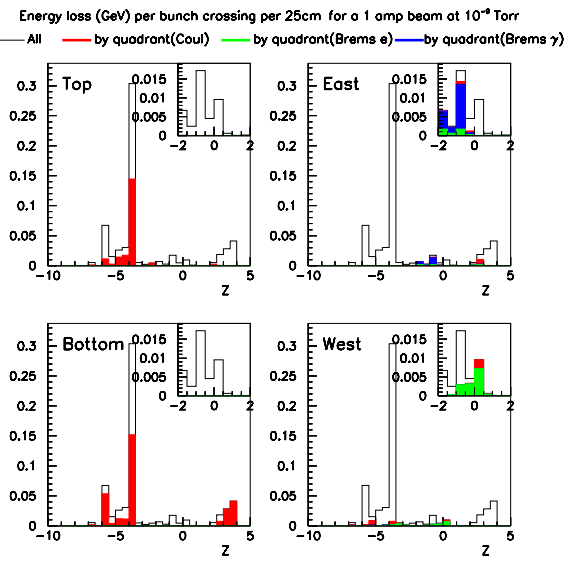

Figure 2: Energy-weighted distribution of beam-gas $e^{-}$ lost in the IR, as a function of hit distance $z_{h}$ from the IP, in four azimuthal quadrants. The insets correspond to the inside of the $\mathrm{BaBar}$ detector $\left(-2<z_{h}<2 \mathrm{~m}\right)$. The vertical scale is in units of $(\mathrm{GeV} / 25 \mathrm{~cm}) \times(\mathrm{A}-\mathrm{nTorr})^{-1}$. The shadings indicate the contributions, in each quadrant, of Coulomb-scattered $e^{-}$'s, and of Bremsstrahlung $e^{- \text {'s }}$ and $\gamma$ 's. The hollow histogram is the sum over all quadrants.

Even though the hit distribution is peaked (Fig. 3), electromagnetic showers (not modelled by LPTURTLE) dilute the energy flow that reaches the active detector.

A study of background sources around the ring (assuming uniform gas pressure) shows that the LER and HER can be divided into a few "background zones" (Fig. 4) within which the distribution of beam-gas scattering locations is nearly uniform. The zone boundaries reflect the focussing properties of the lattice combined with the location of specific bending magnets: in each ring, the arcs constitute a single zone because of the periodicity of their lattice; the detector straights typically consist of three or four zones, depending on the exact layout of S-bend dipoles, optical-

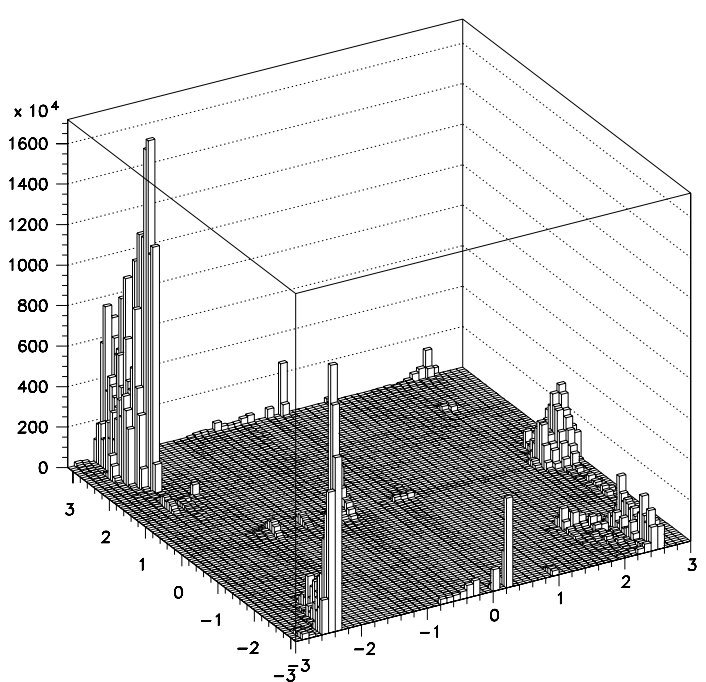

Figure 3: Energy-weighted distribution of beam-gas $e^{+}$ lost in the IR, as a function of hit distance $z_{m}$ (nearest axis, $\mathrm{m}$ ) and azimuth $\phi$ (radians), for a $2.4 \mathrm{~A} e^{+}$beam and a uniform pressure of 1 nTorr.

matching and low- $\beta$ sections. This greatly simplifies the background analysis, as background hits can be reweighted according to the average residual pressure in each zone.

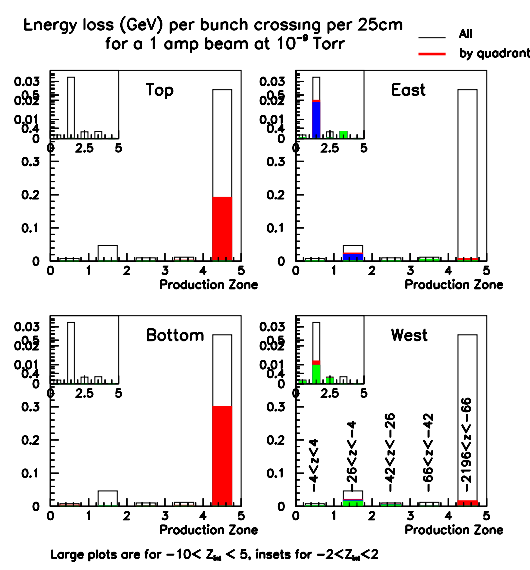

Figure 4: Source distributions of HER beam-gas backgrounds, per quadrant. The longitudinal extent of the background zones is indicated in the bottom-right figure.

\section{RADIATIVE-BHABHA BACKGROUND}

Far off-momentum $e^{+}$and $e^{-}$'s will not follow nominal orbits, but interact with the beam pipe. To evaluate the importance of these effects, radiative Bhabha events have been generated at the IP and the resulting $e^{ \pm} \gamma$ particles, have been followed through the different magnets and aperture limitations within 3 meters in the positron beam direction and 7 meters in the electron side.

The generator [4] provides events with a weight which is transmitted through LPTURTLE. This program gives an accurate description of radiative Bhabha scattering in the very forward direction. Large angle scattering, multi- 
ple photon radiation and 4-lepton processes remain to be studied. At present we have evaluated effects corresponding to the largest fraction of the radiative Bhabha cross section, induced by the interaction of beam particles having radiated a fraction of their energy. The instantaneous dose rate caused by energy-degraded electrons (HER) or positrons (LER) hitting an aperture limitation is shown in Fig. 5 as a function of the primary hit distance $z_{h}$. The magnetic beam-separation scheme again concentrates this background in the horizontal plane.

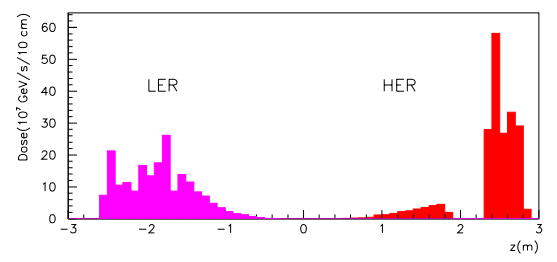

Figure 5: Dose rate impinging on the IR vacuum chamber, and associated with the process $e^{+} e^{-} \rightarrow e^{+} e^{-} \gamma$, normalized to a luminosity of $10^{34} \mathrm{~cm}^{-2} \mathrm{~s}^{-1}$.

\section{COLLIMATION STUDIES}

In the LER, four distant collimators $(\sim 300 \mathrm{~m}$ upstream of the IP) and two nearer ones $<25 \mathrm{~m}$ upstream of the IP have the greatest impact on beam-beam tails. To improve the collimation efficiency and reduce the flux of shower debris, we tightened the distant-collimator apertures and relocated one of the upstream IR collimators. We generate a particle beam that samples all betatron phases in one turn, providing a strategy independent of the shape of the tails, with particles uniformly distributed in position $(x)$ and angle $\left(x^{\prime}\right)$ over ranges defined by $\sigma_{x}$ and $\sigma_{x^{\prime}}$, increased by a factor of 60 to guarantee full sampling. As the LER (unlike the HER) has a large vertical aperture compared to the beam size, only the horizontal plane is important. Fig. 6 depicts the initial phase space distribution of the particle beam generated by LPTURTLE for particles that hit the beam pipe with all collimators left open. Any particle leaving the IP at a larger betatron amplitude will hit the beam pipe long before completing a full turn. The central region is empty as particles pass through unscathed. We generously define the 'background region' as $z_{h}= \pm 25 \mathrm{~m}$ from the IP, and background events are shown as black dots.

Fig. 7 shows the position of background events in the horizontal plane, for an upstream collimator and an equidistant downstream collimator. Tightening the collimator apertures in the distant betatron collimation section reduces the number of particles hitting the beam pipe near the IP. These remaining particles have larger $|x|$ at the downstream collimator position than at the upstream collimator position so the same collimator moved downstream can have a wider aperture, and collimate more particles while producing only harmless secondaries.

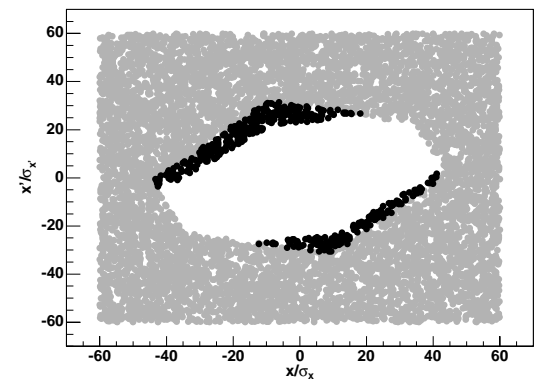

Figure 6: Initial phase-space distribution at the IP. Gray dots: particles lost $>25$ m upstream. Black dots: particles lost within $\pm 25 \mathrm{~m}$ of the IP after one turn.

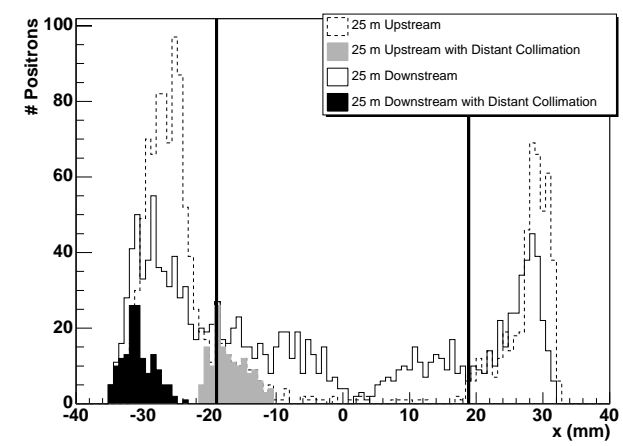

Figure 7: $x$ distributions at near-IP collimators for $e^{+}$'s hitting the beam pipe within $\pm 25 \mathrm{~m}$. The white dashed histogram, $25 \mathrm{~m}$ upstream of the IP, is reduced to the grey histogram if the distant collimators are set to $10 \sigma$. The solid white and black histograms are for $25 \mathrm{~m}$ downstream. The vertical lines correspond to typical collimator settings.

\section{CONCLUSIONS}

The LPTURTLE program provides a good description of background processes at PEP-II, and is a useful tool for understanding their origins and for their reduction.

We would like to thank Mike Sullivan and Cathy Carr for their careful work in ascertaining the precise co-ordinates of the apertures in the beam pipe near the Interaction Point.

\section{REFERENCES}

[1] J. T. Seeman et al., 'Results and Plans of the PEP-II B factory', these Proceedings.

[2] B. Aubert et al., 'The BaBar detector', Nucl. Instrum., \& Meth. A479 1 (2002)

[3] T. Fieguth et al, 'Modeling Lost-Particle Backgrounds in PEP-II Using LPTURTLE', These proceedings, TPPP025.

[4] R. Kleiss and H. Burkhardt, Comput. Phys. Commun. 81 (1994) 372. 